\title{
Esfero rojo, esfero azul: Un enfoque tradicional de la educación actual en el Ecuador
}

\section{Red corner, blue corner: A traditional approach to current education in Ecuador}

\author{
Miguel Angel Solís Cevallos \\ msolis4161@pucem.edu.ec \\ Pontificia Universidad Católica del Ecuador, Portoviejo \\ Ecuador \\ https://orcid.org/0000-0003-2338-3422 \\ Esthela María San Andrés Laz \\ esthelasanandres@gmail.com \\ Universidad Técnica de Manabí, Portoviejo \\ Ecuador \\ https://orcid.org/0000-0003-3462-8528
Marcos Fernando Pazmiño Campuzano mpazmino@utm.edu.ec
Ecuador
https://orcid.org/0000-0002-9534-2059 \\ Universidad Técnica de Manabí, Portoviejo
}

Recibido: 29 de septiembre de 2019

Aprobado: 30 de octubre de 2019

\begin{abstract}
RESUMEN
En el presente artículo se analiza la educación actual en el Ecuador desde un enfoque tradicional para conocer si durante el proceso de construcción del conocimiento, todavía existen prácticas de enseñanza tradicionales y si se lograba el propósito de consolidar capacidades en los estudiantes. Esta investigación estuvo sustentada con la revisión de fuentes bibliográficas mediante el método exploratorio de análisis documental. Para la recolección de información se utilizó como instrumento, la encuesta, que estuvo dirigida a docentes de la Unidad Educativa Fiscal "Uruguay" de la ciudad de Portoviejo, Ecuador, utilizando para la muestra de la población a consultar la fórmula de cálculo de Murray y Larry. Los resultados obtenidos permitieron determinar que las instituciones y docentes a pesar del avance tecnológico y un currículo que en teoría fomenta la adquisición de habilidades, destrezas y competencias para que puedan desarrollar un pensamiento lógico y crítico, estos
\end{abstract}


mantienen prácticas de enseñanza y aprendizaje con modelo pedagógico tradicional en donde no se logran las competencias básicas, la cual se ve reflejado en la educación superior y en el empleo.

Descriptores: Modelo pedagógico; Realidad educativa; Competencias.

\begin{abstract}
This article analyzes the current education in Ecuador from a traditional approach to know if during the process of knowledge construction, there are still traditional teaching practices and if the purpose of building skills in students was achieved. This research was supported by the review of bibliographic sources through the exploratory method of documentary analysis. For the collection of information, the survey was used as an instrument, which was addressed to teachers of the Fiscal Education Unit "Uruguay" of the city of Portoviejo, Ecuador, using the sample to calculate the Murray calculation formula and Larry The results obtained allowed us to determine that institutions and teachers despite technological progress and a curriculum that theoretically promotes the acquisition of skills, abilities and competences so that they can develop logical and critical thinking, they maintain teaching and learning practices with a pedagogical model traditional where basic skills are not achieved, which is reflected in higher education and employment.
\end{abstract}

Descriptors: Pedagogical model; Educational reality; skills.

\title{
INTRODUCCIÓN
}

Es típico escuchar en los estudiantes una vez que el docente empieza un nuevo tema o un nuevo párrafo de una clase, "profesor se escribe con rojo o con azul" refiriéndose al color del esfero que en ese momento necesita utilizar, además es común también oír por parte de docentes y profesionales "la educación de antes era mejor", si estas situaciones se ubican en el tiempo, son frases que se las viene manifestando durante más de 50 años, pero esto, no solo queda en lo retorico; sino que da la pauta para expresar un análisis general de lo poco o nada que la enseñanza educativa ha cambiado desde finales del siglo XIX.

La educación como un hecho y practica social, se reserva al desarrollo de la capacidad intelectual, afectiva y moral de un individuo, la cual se manifiesta dependiendo de la cultura y normas de convivencia social a la que se pertenece (Padilla \& Ortiz, 2018), siendo el principal beneficiado el alumno. Pero es importante 
preguntarse ¿Qué tanto influye el modelo de enseñanza pedagógica utilizado para lograr este propósito?, conociendo los continuos avances tecnológicos que en concordancia con los postulados sobre educación de la (UNESCO, 2017), dónde buscan fortalecer las capacidades, habilidades y conocimientos humanos mediante una educación de calidad.

La práctica educativa en los procesos de enseñanza - aprendizaje, en muchos casos no cambia, puesto que los docentes siguen reproduciendo métodos pedagógicos tradicionales, no acordes a las necesidades y potencialidades que requiere la educación actual (Dávila, 2017), aunque los programas y objetivos actuales pretenden modelos formativos orientados hacia el constructivismo.

En ese contexto, en el Ecuador y en América Latina, a partir de 1950, si bien se dieron logros importantes como el de ir incorporando al sistema educativo a niños y jóvenes de los estratos sociales más bajos, que normalmente estaban marginados, el acceso de mujeres, grupos indígenas y en la actualidad a grupos de condición especial, pero al mismo tiempo se acumulaban problemas, no sólo de carácter cuantitativo, sino cualitativo en donde solo se estaban privilegiando contenidos y resultados de aprendizajes como un todo.

En este proceso de cambios, el Ministerio de Educación del Ecuador (MEE, 2016), pregona la aplicación de estándares de calidad por medio de un ajuste curricular implementado para la educación básica general y bachillerato, en donde los estudiantes deben adquirir habilidades y destrezas para que puedan desarrollar un pensamiento lógico y crítico y que estén en capacidad de aportar solución a las diferentes problemáticas que presenta la sociedad actual, constructivismo puro. En concordancia con estos preceptos es necesario revelar que no hay mecanismos para evaluar si el conocimiento adquirido por los estudiantes es realmente útil para la vida diaria, (Delval, 2013).

En realidad están dadas las condiciones para una educación de calidad, con el avance de la tecnología para sostener la formación de competencias basada en la investigación poniendo en práctica la teoría expuesta en el proceso de enseñanza y aprendizaje. Pero el complemento entre los modelos educativos y la tecnología está 
en compás de espera, porque los docentes que se gradúan están aplicando métodos tradicionales, no acorde al estilo de aprendizaje de los estudiantes que son diferentes a los de otra época ni a las necesidades de la sociedad actual.

El aporte que brinda la presente investigación es conocer, lo que es modelo pedagógico de enseñanza- aprendizaje tradicional, identificar sus ventajas y desventajas, analizar en el contexto de la educación actual, en especial dar una mirada hacia la realidad del país, desde el ámbito educativo con relación al empleo, pretende además comprender porque no se logra consolidar un nuevo modelo educativo, sino que al final se recurre siempre a las prácticas de enseñanza educativas tradicionales.

\section{MATERIALES Y MÉTODOS}

El presente artículo tomó en cuenta el método exploratorio como parte relevante de la investigación en la que se empleó la técnica del análisis documental para lo cual se revisaron bibliografías que definen los conceptos más destacados sobre método de enseñanza tradicional en la educación actual.

La encuesta fue el instrumento utilizado para la recolección de información y estuvo dirigida a docentes de la Unidad Educativa Fiscal "Uruguay" de la ciudad de Portoviejo, Ecuador, utilizando la fórmula de cálculo de la muestra de una población presentada por (Murray \& Larry, 2009).

Los principales temas que se abordaron y en la que se sustenta esta investigación son:

- Las prácticas de enseñanza que utilizan los docentes de hoy en día

- El sistema educativo alienta el aprendizaje constructivista pero con modelo tradicionalista

- La relación entre el perfil de salida del estudiante de secundaria con lo que la universidad y las profesiones requieren 


\section{ANÁLISIS Y DISCUSIÓN DE LOS RESULTADOS}

\section{Modelo de educación tradicional}

Un modelo es la imagen o representación de un conjunto de relaciones que definen un fenómeno, (Flórez, 1994), el mismo que se debe reconocer las bases sobre la que fue construido el fenómeno estudiado, así de esta manera se podrá analizar en contexto los alcances que este paradigma tiene facilitando su comprensión, (De Zubiria, 2010).

En referencia, un modelo educativo la podemos considerar como una recopilación de diferentes teorías y enfoques pedagógicos que sirven para orientar a los docentes al momento de construir los programas de estudio y la sistematización en el proceso de enseñanza y aprendizaje. Los modelos educativos representan la forma en que el conocimiento debe ser impartido, por lo tanto pueden ser consideradas como orientaciones pedagógicas, así como teorías, además de técnicas que van acordes al desenvolvimiento de los docentes, el enfoque epistemológico asumido en la estructuración del currículo, percibe el modo de actuar docente en su praxis profesional, así pueden trabajar desde una noción conductista, constructivista, socio crítico, tal como indica Aldana Zavala (2019).

El construir un modelo educativo implica la transformación del método pedagógico de una institución educativa así como sus métodos de enseñanza; debe estar en coherencia y de acuerdo al paradigma que la institución visualiza y con la organización académica que esta pretenda, implica además que todos los miembros de la comunidad educativa participen y tengan la decisión y voluntad de aceptar un modelo que se ajuste a las necesidades que se requiera, el cual no tiene que ser una decisión vertical por parte de las autoridades, debe ser un consenso por el bienestar académico del alumno, así mismo se requiere involucrar en la formación de los nuevos docentes, una visión transformadora de la educación, tal como indican Romero \& Villasmil (2017).

En tal sentido, un modelo pedagógico es la representación de las relaciones que predominan en el fenómeno de enseñar, considerado como un paradigma, que puede coexistir con otros paradigmas en torno a la pedagogía y organizar un sistema con la 


\section{Revista Arbitrada Interdisciplinaria KOINONIA \\ Año IV. Vol IV. N8. Julio - Diciembre 2019 \\ Hecho el depósito de Ley: FA2016000010 ISSN: $2542-3088$ \\ FUNDACIÓN KOINONIA (F.K). Santa Ana de Coro. Venezuela.}

Miguel Angel Solís Cevallos; Esthela María San Andrés Laz; Marcos Fernando Pazmiño

práctica educativa (Florez \& Tobon, 2001). Los modelos pedagógicos durante el desarrollo de una clase deben de partir de vivencias y experiencias concretas ya que supone que lo más cercano, lo más próximo y concreto es así mismo lo más fácil de conocer, (De Zubiria, 2006), aunado a lo planteado por lanni Gómez (2017), quien destaca que la educación debe recuperar su rol de orientación integral para promover las bases de una formación no tradicional.

Dentro de un modelo educativo la relación central o el vínculo más estrecho se da entre docente y alumno, inclusive afectivo y emocional en ocasiones, pero es prioritario para que den las condiciones para buena enseñanza y un buen aprendizaje sobre todo el desarrollo de habilidades socioemocional, El modelo pedagógico, se lo concibe bajo un método que busca interrelacionar los agentes básicos de la comunidad educativa con los conocimientos científicos para conservarlo, producirlo o recrearlo dentro de un contexto histórico, geográfico y cultural determinado (Vásquez, 2013), por lo que un modelo educativo debe ser inspirador, con ideas claras y precisas de lo que quiere y pretender lograr, definir y justificar la elección de contenidos, los conocimientos que se van impartir y las habilidades y destreza que se pretender desarrollar, todo esto en función del tipo de persona que vayamos a insertar a la sociedad, con pensamiento propio, crítico y lógico.

Las instituciones educativas funcionan como un sistema por lo que el modelo educativo no debe estar circunscrito a los contenidos, programas y planes de estudio, sino alcanza el funcionamiento acorde a sus objetivos. El modelo educativo no debe estar en contraposición con el proceso de desarrollo. Es indispensable que en cualquier oferta educativa debe contener al menos tres elementos básicos: Primero, el objetivo de la oferta académica, es decir qué tipo de alumnos quiere la institución formar. Segundo, que conocimientos y habilidades se van a brindar a los alumnos para que vayan en función del objetivo. Tercero, como complementar la enseñanza que les falta a los alumnos para lograr el desarrollo integral como individuos, con su propia identidad y conducta.

Sin embargo para perfeccionar esta oferta educativa, es primordial cualificar al alumno y al docente que vamos a seleccionar, la logística necesaria como bibliotecas y 


\section{Revista Arbitrada Interdisciplinaria KOINONIA \\ Año IV. Vol IV. N8. Julio - Diciembre 2019 \\ Hecho el depósito de Ley: FA2016000010 ISSN: $2542-3088$ \\ FUNDACIÓN KOINONIA (F.K). Santa Ana de Coro. Venezuela.}

Miguel Angel Solís Cevallos; Esthela María San Andrés Laz; Marcos Fernando Pazmiño

laboratorios, la impartición de disciplina no visto como conducta sino como orientación que los haga madurar con libertad, valores, conductas cotidianas, capacidades cognitivas y socioemocionales, elementos como el trato de las autoridades y personal administrativo, son parte de esta contribución para formar un alumno modelo.

Los modelos pedagógicos que han contribuido a la educación, sin considerar cual sería el perfecto para ser utilizado como estándar, son el modelo pedagógico tradicional el cual se enfoca el presente artículo, el modelo conductista muy relacionado al tradicional, modelo de escuela de nueva y el constructivista.

Se puede considerar a Juan Comenio como el padre del modelo pedagógico tradicional que en el siglo XVI, basaba su teoría en que cada área del conocimiento lo abarca como un conjunto completo, que vendría a ser un nuevo punto de partida para otro, mucho más alto, profundo y extenso, esta formación debe empezar desde los principios más elementales del aprendizaje para la adquisición de un conocimiento más universal, (Vergara y Cuentas, 2013). Más adelante en el siglo XVIII empieza a gestarse con el surgimiento de la escuela como institución, logra la relevancia con el advenimiento de la pedagogía como ciencia en el siglo XIX, que se caracterizaba porque los contenidos en la que se basaba la enseñanza eran los conocimientos y valores acumulados por la humanidad los que se transmiten por el maestro como verdades absolutas desligadas del contexto social e histórico en que el alumno se desenvuelve. (Rodríguez, 2013).

El desarrollo de las ciencias trajo consigo el uso de nuevas tecnologías para ser aplicadas en la producción en masa que trajo una transformación económica, social y educativa conocida como Revolución industrial. Los sistemas educativos se crearon para responder a los intereses empresariales de la época, las competencias en matemáticas, ciencias y lenguaje eran imprescindibles para las economías industriales mientras que la universidad dejo de lado cualquier actividad que implique el alma, el cuerpo y el uso de los sentidos y gran parte del cerebro, (Robinson, 2009). Este modelo fue construido sobre el modelo de fábrica, la educación general enseñaba los fundamentos de lectura, escritura y aritmética. Esto era el denominado "programa descubierto". Pero la realidad es que existía un llamado "programa 


\section{Revista Arbitrada Interdisciplinaria KOINONIA \\ Año IV. Vol IV. N8. Julio - Diciembre 2019 \\ Hecho el depósito de Ley: FA2016000010 ISSN: $2542-3088$ \\ FUNDACIÓN KOINONIA (F.K). Santa Ana de Coro. Venezuela.}

Miguel Angel Solís Cevallos; Esthela María San Andrés Laz; Marcos Fernando Pazmiño

encubierto" más elemental. Este programa se ejecutaba para tres parámetros: puntualidad, obediencia trabajo mecánico y repetitivo. El trabajo de fábrica exigía obreros que llegasen a sus labores a una hora puntual, trabajadores que aceptasen órdenes sin discusión y exigía hombres y mujeres para trabajar como esclavos en máquinas y oficinas realizando operaciones mecánicas y repetitivas, (Toffer, 1985), por esto se educaba niños y adolescentes predispuestos a la sumisión, obediencia y cumplimiento de tareas en cualquier momento y en silencio.

En este sentido la función de las instituciones educativas era las transmisión de saberes específicos, las valoraciones y normas culturales socialmente aceptada además de los contenidos técnicos, (De Zubiria, 2006) lo cual sustentaba y mantenía la cultura y esta se traslada de generación en generación en donde el aprendizaje basado en contenidos tenían un orden establecido de una forma en que un nuevo tema solo se podía enseñar solo cuando el anterior era previamente aprendido mediante la memorización.

\section{Características del modelo pedagógico tradicional}

En este modelo educativo el docente es el sujeto del proceso de enseñanza y aprendizaje, tiene una estructura vertical en donde el profesor impone reglas y normas, elige los contenidos y planes de estudio que él cree son los que el alumno necesita para su educación. Este modelo plantea una diferenciación entre el lugar que ocupa el docente como aquel que imparte el conocimiento y el lugar que ocupa el alumno como aquel que recibe la enseñanza.

Al alumno se lo preparaba para ser un modelo o representación de alguien más en la historia, es ahí donde el docente empezó a ser el centro de ese proceso. El papel del docente era repetir y repetir hasta que el alumno aprendiera de memoria, se limitaba a imitar y copiar, porque se consideraba que con la reiteración el conocimiento era adquirido de forma significativa. Este modelo fue concebido para formar jóvenes con actitudes de obediencia, sumisión y cumplimiento que para momento histórico era valedero.

Su objetivo, sin embargo es que el alumno aprenda, mediante la transmisión del conocimiento directo del docente, reconoce la memorización como método de 
aprendizaje, le da énfasis a la información por encima del concepto y la esencia de la información. A continuación se detallan las principales características de este modelo pedagógico:

El docente es quien posee y es el único transmisor de conocimientos

Propone el docente que, cuando y como enseñar

El alumno es un ente pasivo receptor del conocimiento

El alumno no tiene derecho a opinar sobre un contenido

El currículo es un plan obligatorio de contenidos

La evaluación es cuantitativa, final y se la realiza mediante exámenes muy rigurosos para determinar cuánto el alumno ha asimilado los conocimientos La disciplina es severa para garantizar un ambiente adecuado para el aprendizaje

El estudiante debe aprender de manera memorística, el cual debe poseer una capacidad alta de percepción

No existen metodologías de trabajo ni recursos didácticos para la labor docente Los contenidos contienen información técnica y socialmente aceptadas

Se privilegia la transmisión de conocimientos y de valores, básicos para la socialización y competencias mínimas para que el alumno se desenvuelva en el mundo laboral

No estimula la investigación ni la creatividad, tampoco fomenta las capacidades críticas ni el razonamiento. (Vergara y Cuentas, 2015)

\section{Realidad de sistema educativo actual}

Mientras el mundo de la comunicación, información y tecnología avanza en forma acelerada y que debería estar al servicio de la educación o por lo menos usarla como herramienta para mejorar el proceso de enseñanza - aprendizaje, el sistema está planteado como una pirámide de arriba hacia abajo estando los docente en lo más alto y los alumnos en lo más bajo, método implementado desde el siglo XIX, (Calleja, 2010). El sistema educativo actual no ha variado a lo largo del tiempo, un docente 


\section{Revista Arbitrada Interdisciplinaria KOINONIA \\ Año IV. Vol IV. N8. Julio - Diciembre 2019 \\ Hecho el depósito de Ley: FA2016000010 ISSN: $2542-3088$ \\ FUNDACIÓN KOINONIA (F.K). Santa Ana de Coro. Venezuela.}

Miguel Angel Solís Cevallos; Esthela María San Andrés Laz; Marcos Fernando Pazmiño Campuzano

entra a la clase, habla, los alumnos toman notas y como no pueden recordar los temas que se les converso, les toman exámenes, pero una vez dados, se olvidan de todo, (Manzano, 2007). El objetivo es aprobar exámenes y tener buen promedio en notas, sin importar el después.

La educación debe ayudar a cada persona a encontrar su lugar en la sociedad. La educación bajo reglas estrictas no ha tenido los resultados esperados por la comunidad porque no es capaz de identificar los estilos y necesidades de aprendizaje del alumno así como desarrollar sus aptitudes y habilidades individuales. (Gerver, 2010).

La escuela, luego de la familia es una entidad necesaria para la formación de la persona, pero esta no incentiva a la creatividad ni al pensamiento lógico y crítico. A los docentes les interesa que los alumnos aprendan temas de contenidos previamente asignados y que se sigan al pie de la letra como imposición porque supuestamente el adiestramiento es el camino que debe seguir para desenvolverse en la vida. En cada una de las actividades en clases deben seguir una ruta establecida por el maestro, ajustado tal como lo pretende él y los textos escolares, limitando el potencial de inteligencia que puedan tener los estudiantes, haciéndolos inclusive más torpes por no dejarlo usar su imaginación, (Rius, 2012).

Bajo esta perspectiva, la educación en Ecuador promueve desde su marco legal el modelo constructivista. La Constitución De la república del Ecuador (2008) en el artículo 343 establece un sistema de educación que tiene como finalidad el desarrollo de capacidades y potencialidades individuales y colectivas, que generen el aprendizaje significativo por medio del conocimiento, el que tendrá como centro al sujeto que quiere aprender. Este sistema debe ser dinámico, flexible, incluyente eficiente y eficaz.

Por tanto, la educación concebida como un factor de cambio, debe permitir a los alumnos apropiarse de su ser social en su comunidad, alcanzando habilidades y aptitudes que proporciones la capacidad de diagnosticar problemas, al mismo tiempo formular alternativas para encontrar una solución a ese fenómeno, además de vivir en forma armónica, en donde pueda articular la teoría con la práctica mediante el 


\section{Revista Arbitrada Interdisciplinaria KOINONIA \\ Año IV. Vol IV. N8. Julio - Diciembre 2019 \\ Hecho el depósito de Ley: FA2016000010 ISSN: $2542-3088$ \\ FUNDACIÓN KOINONIA (F.K). Santa Ana de Coro. Venezuela.}

Miguel Angel Solís Cevallos; Esthela María San Andrés Laz; Marcos Fernando Pazmiño

raciocino lógico y critico asumiendo responsabilidades en todo el proceso de desarrollo integral y autónomo de su entorno interno y externo, (Ministerio de educación de Ecuador, 2016).

En ese sentido, por parte del Ministerio de educación del Ecuador se implementan estándares de calidad educativa y de ajuste curriculares en lo que se presume que el estudiante adquiere conocimientos desarrolla destrezas sobre situaciones concretas en contextos diferentes para resolver problemas, al mismo tiempo la Universidad presupone que esos mismos alumnos están listo para desarrollar competencias que son indispensables en ese nivel educativo y más adelante en su vida profesional, (Barragán \& Ortega, 2017) lo que queda en duda cuando es notorio que no se cuentan con las herramientas necesarias y que los métodos implementados en la enseñanza fueron los adecuados y necesarios para poner en práctica lo asimilado.

Si bien es cierto lo que se pretende con la constitución, leyes y reformas que deben ser aplicadas en las instituciones escolares del Ecuador, existe un desconocimiento de que técnicas y estrategias se deben utilizar con base en las nuevas tecnologías, limitando métodos de enseñanza tradicional que imposibilita a los estudiantes acceder al conocimiento y estén en capacidad de competir en un mundo cada vez más globalizado que se evidencian en el rendimiento académico cuyo aprendizaje no es el adecuado. Todo lo propuesto se línea con la corriente pedagógica constructivista, pero en la práctica no se logran superar viejas prácticas tradicionales, maltratadoras y descontextualizadas, (López, 2011).

Para corroborar si los docentes y las instituciones mantienen prácticas de enseñanza con modelo pedagógico tradicional se realizó encuesta a docentes de la Unidad Educativa Fiscal "Uruguay" de la ciudad de Portoviejo, Ecuador. Institución emblemática que cuenta con 2400 estudiantes y 100 docentes de planta. Para calcular el tamaño de la muestra se consideró a la población de maestros en la cual se utilizó la siguiente formula de Murray y Larry:

$$
n=\frac{N \sigma^{2} Z^{2}}{(N-1) e^{2}+\sigma^{2} Z^{2}}
$$

Donde:

$\mathrm{n}=$ Tamaño de muestra. 
$\mathrm{N}=$ Población .

$\sigma=$ Desviación estándar de la población que, cuando no hay valor, se utiliza un valor constante de 0,5 .

$Z=$ Nivel de confianza. Es un valor constante, si no se tiene un valor, se toma un $95 \%$ que equivale a 1,96 (más usual).

e = Límite de error muestral, se utiliza un valor entreel $1 \%(0,01)$ y $9 \%(0,09)$, normalmente 0,05 .

En la tabla 1 se muestran los resultados sobre las características de modelo tradicional y si están se aplican actualmente en las instituciones

Tabla 1

Prácticas de enseñanza tradicional

\begin{tabular}{|c|c|c|c|c|}
\hline & \multicolumn{2}{|l|}{ Si } & \multicolumn{2}{|l|}{ No } \\
\hline & cantidad & $\%$ & cantidad & $\%$ \\
\hline Utiliza los textos escolares que brinda el Ministerio & & & & \\
\hline de Educación para el desarrollo de sus clases & 75 & 93,75 & 5 & 6,25 \\
\hline Considera que es obligatorio cumplir con los & & & & \\
\hline contenidos de los textos escolares & 46 & 57,5 & 34 & 42,5 \\
\hline $\begin{array}{l}\text { Las tareas enviadas a los estudiantes deben ser } \\
\text { presentadas tal como usted la pidió }\end{array}$ & 60 & 75,0 & 20 & 25,0 \\
\hline Las evaluaciones son cuantitativas mediante & & & & \\
\hline exámenes & 64 & 80,0 & 16 & 20,0 \\
\hline Los pupitres están ubicados normalmente en filas y & & & & \\
\hline columnas & 68 & 85,0 & 12 & 15,0 \\
\hline $\begin{array}{l}\text { El Ministerio de educación brinda herramientas } \\
\text { tecnológicas y materiales didácticos para una mejor }\end{array}$ & & & & \\
\hline labor docente & 16 & 20,0 & 64 & 80,0 \\
\hline $\begin{array}{l}\text { Cree que el sistema educativo promueve el } \\
\text { desarrollo del talento individual de cada alumno, de }\end{array}$ & & & & \\
\hline lo que más le apasiona & 10 & 12,5 & 70 & 87,5 \\
\hline
\end{tabular}


Es común observar como a pesar de los avances en la ciencia y en la tecnología, teniendo estudiantes con otra manera de pensar y actuar diferentes a otras épocas, el modelo de pedagogía tradicional todavía está presente, pues desde que se ingresa a una aula de clases la forma como están ubicadas las bancas o los pupitres no ha cambiado desde hace más de 200 años, docentes que continúan enseñando como si fueran el centro de atención, se creen la máxima autoridad y quienes poseen solo el conocimiento, siendo los alumnos meros receptores de ese supuesto aprendizaje. Gráfico 1

Asimilación del aprendizaje

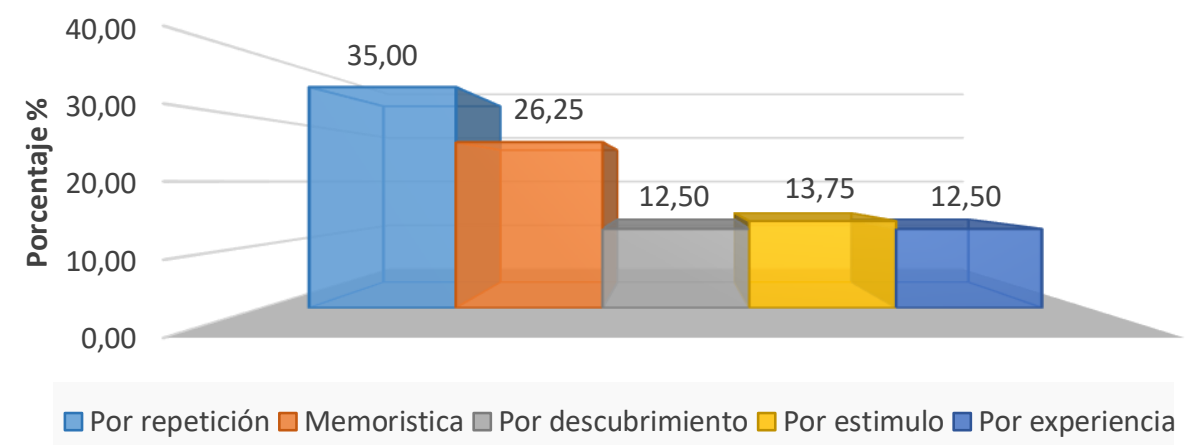

Tal como muestran los resultados de la figura 1, los estudiantes aún continúan reteniendo conocimientos de forma tradicional por repetición y memorística. El docente actual cuando imparte su clase, lo realiza en forma de monologo, repitiendo contenidos de un currículo existente desde 1996, que aunque busquen el desarrollo de destrezas con criterios de desempeño, no hay un cambio real en el proceso (El ciudadano, 2011) y como se visualiza no da oportunidad al desarrollo de la investigación.

El contenido curricular además de académico es racionalista, que tiene un apego con la ciencia, con lógica interna en diferentes situaciones, conllevando al perfeccionamiento de pensamientos empíricos, descriptivos, no teóricos, (Ortiz, 2013), contenidos que deben ser reescritos a un cuaderno considerado ideal en donde los títulos deben ser señalados con esfero rojo y los conceptos con esfero azul, 
luego al pie de la letra exponer o comprobar lo aprendido en exámenes rigurosos que solo buscan una nota cuantitativa, más no cualitativa.

\section{Relación de modelo pedagógico tradicional con empresa actual}

La ciencia y la tecnología avanzan en forma dinámica, la empresa es una de las que más se beneficia de esos cambios, explotando cada uno de esos recursos y herramientas para mejorar la productividad técnica. Pero qué se puede decir del recurso humano si la educación no ha mejorado al ritmo en que la sociedad en general lo hace. La educación tradicional solo brinda conocimientos en forma vertical docentealumno sin el apoyo que puede brindar las herramientas tecnológicas, más aun el objetivo es crear alumnos memorísticos sin capacidad de actuar por sí solo, cuando lo que más espera la empresa es contar con personas creativas, flexibles, capaces de adaptarse a situaciones nuevas, trabajo en equipo, con criterio propio, muy alejado de lo que brinda la educación tradicional.

Gráfico 2

Actividades constructivistas que estimulen el aprendizaje

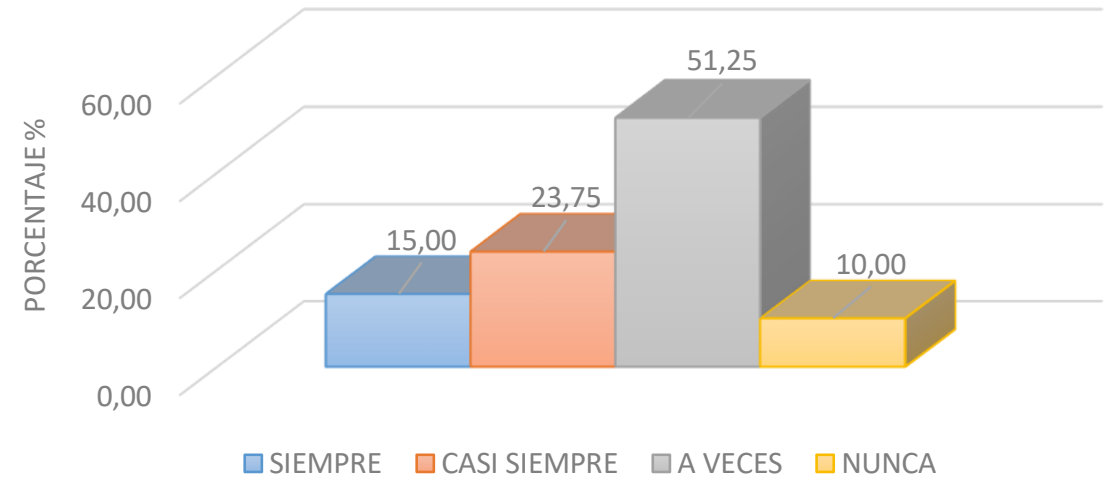

Se puede comprobar los resultados de la figura 2 que las actividades que desarrollan en clases los docentes en un alto porcentaje no facilitan la creatividad, no promueven 


\section{Revista Arbitrada Interdisciplinaria KOINONIA \\ Año IV. Vol IV. N8. Julio - Diciembre 2019 \\ Hecho el depósito de Ley: FA2016000010 ISSN: $2542-3088$ \\ FUNDACIÓN KOINONIA (F.K). Santa Ana de Coro. Venezuela.}

Miguel Angel Solís Cevallos; Esthela María San Andrés Laz; Marcos Fernando Pazmiño

la investigación ni usan la tecnología como apoyo al proceso de enseñanza aprendizaje.

En teoría, se están dando grandes cambios en los currículos educativos, pero en la práctica, la enseñanza sigue siendo tradicional. Un currículo debe ser sólido, fundamentado, coherente, técnico y ajustado a las necesidades de aprendizaje de una sociedad determinada, que debe tener los recursos suficientes que garanticen las condiciones mínimas para el sostenimiento, continuidad y concreción de las intenciones educativas de un estado y que deben fortalecer procesos, programas de enseñanza y aprendizaje de calidad que guarde relación con las necesidades individuales y grupales, (Ministerio de educación de Ecuador, 2016)

El currículo es un instrumento de una política educativa de un proyecto global de la enseñanza y que está orientado por un proyecto educativo más amplio inspirado por la visión del hombre por la búsqueda de una cultura ciudadana. Los currículos deben tener por objetivo principal identificar, definir y estructurar los contenidos escolares de la información y documentación con miras a la transmisión y construcción por parte de los alumnos de saberes operacionales, (Gómez, 2017).

El propósito del currículo basado en destrezas y competencias en el sistema educativo ecuatoriano, es que esté acorde al perfil de salida en lo profesional como respuesta a la demanda ocupacional con dominio en ciencia y tecnología, con base solidad en valores, éticos y cívicos que le permito adquirirlo por medio del aprendizaje producto del estudio o de la práctica de un determinado contenido y que con buen criterio lo afirma Pozo (2014) en el que manifiesta que el aprendizaje humano es un proceso de reflexión individual que permite cambiar la forma en que vemos el mundo, de representarnos por sí mismo y a los demás.

Si en la revolución industrial el rol de la educación era prepararlos para el trabajo, en la actualidad ¿Habrá cambiado eso? El currículo educativo se mantiene desde hace más de dos siglos con asignaturas consideradas básicas. Matemáticas, Ciencias y Lenguaje; sin duda que son necesarias, pero ¿Se le da dado el enfoque holístico o multidisciplinar?, por medio de proyectos investigativos o con aplicación de tecnologías. ¿Por qué aún son imprescindibles?, sin duda la mayoría de las 
ocupaciones actuales todavía se sustentan en el conocimiento y las competencias que se derivan de ellas.

\section{Gráfico 3}

Educación con enfoque interdisciplinar

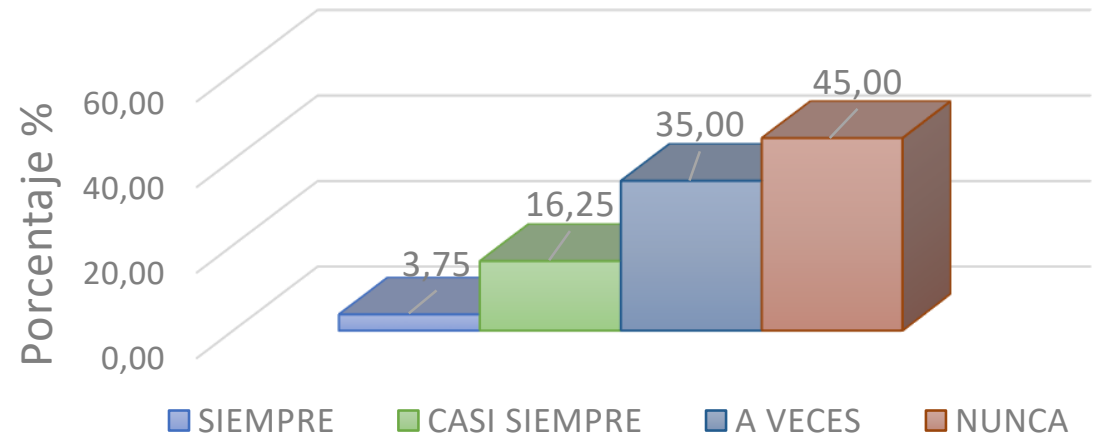

El Ministerio de Educación del Ecuador (2016), pretende que la educación tengan carácter interdisciplinar, para esto deben las instituciones realizar proyectos que abarquen varias asignaturas, pero como arrojan los resultados de la figura 3, esto por lo general se lo realiza algunas veces o no se da nunca.

El sistema educativo actual guarda todavía una estrecha relación con las necesidades sociales, que es el fin mismo de la educación. Sin embargo como pasaba en la época anterior estas exigencias de la sociedad imponen el modelo educativo y que este tenga un carácter clasista que respondan a la formación del individuo como un ente preparado para el ámbito económico-social, es decir que la educación responde a la ideología de la clase dominante que intenta mantenerse en el poder, aunque existen sectores progresistas que luchan por transformar la educación para que contribuya al verdadero progreso social, (Ortiz, 2011).

En este sentido existe una direccionamiento a seguir con el statu quo, según Educación Ecuador (2018) las profesiones más demandadas por los estudiantes para continuar sus estudios universitarios en los últimos años y cuya tendencia se mantendrá en el 2019 son; Medicina y enfermería, Administración de Empresa, 
Ingeniera civil, Arquitectura, Contabilidad y Auditoría, Economía, Comunicación Social, Psicología clínica, Derecho, Odontología, Ingeniería comercial, Industrial, Agronomía. Como se puede notar, aun en los actuales momentos las asignaturas básicas son el complemento de la labor profesional.

Desde el punto de vista del empleo, es decir en que se ocupan las personas que demandan las profesiones anotadas en el párrafo anterior se muestra en el grafico siguiente:

\section{Gráfico 4}

Distribución del mercado laboral por actividad económica
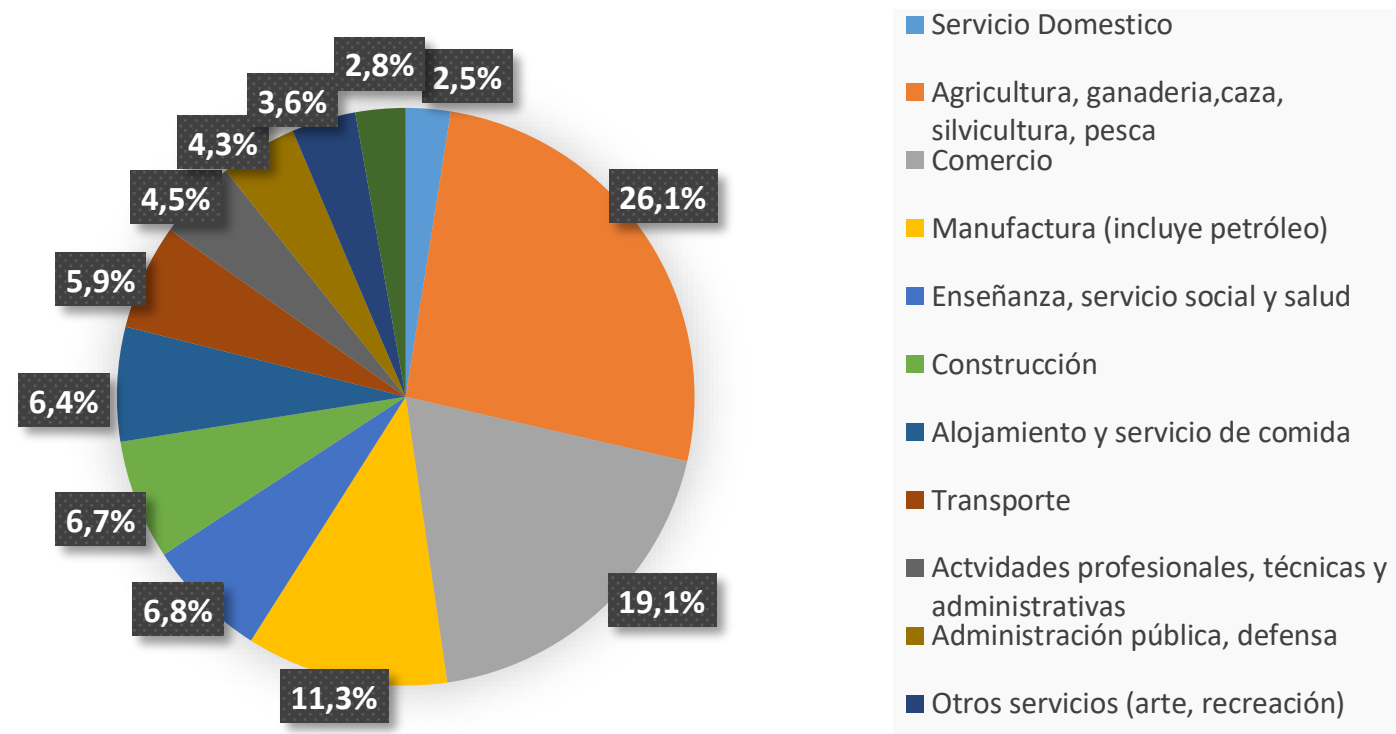

Fuente: INEC (2018)

Como contraste a las carreras demandadas por parte de los estudiantes en la universidad con el porcentaje de empleo al cual realmente va dirigida la ocupación, se puede notar que lo que brinda la educación no va en concordancia con el empleo que de esta se debe generar, solo un poco más del 50\% complementa teoría con la práctica, estudio con trabajo y que termina sustentando en la figura 5. 


\section{Gráfico 5}

Grado de educación de la población empleada

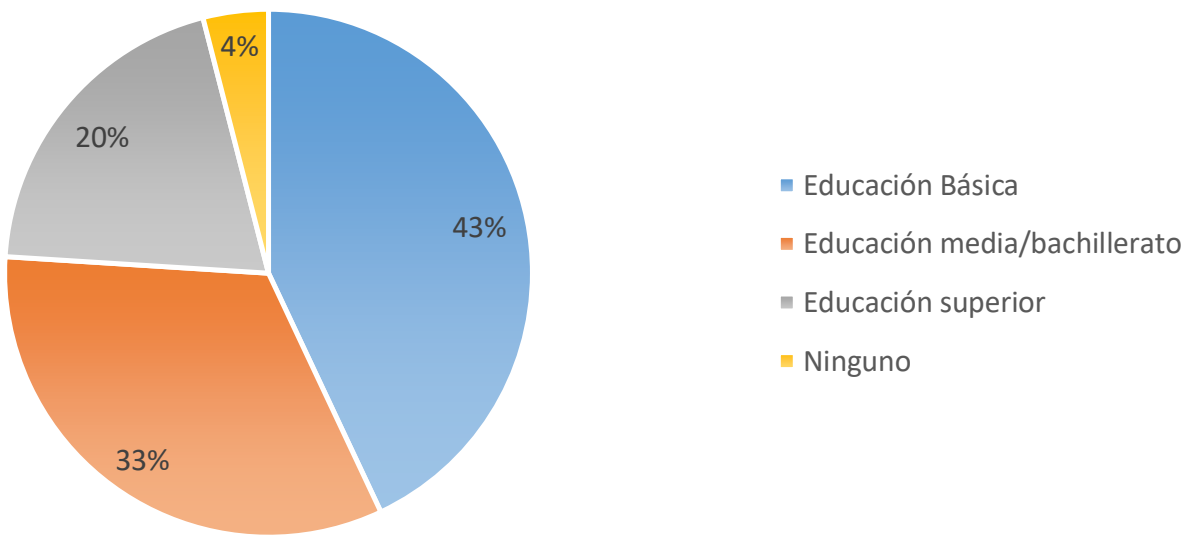

Fuente; INEC (2018)

El mayor porcentaje de la población ocupada coincide con el nivel de educación, lo que es preocupante conociendo que la mayoría son jóvenes con deberían tener una instrucción calificada y superior. Inclusive aun teniendo instrucción superior según el INEC (2018) por medio de un estudio realizado por el Grupo Adecco el 57\% de jóvenes con nivel universitario está desempleado o se dedicaron a otra actividad ajena a su instrucción.

Un mercado laboral debe ser análogo con el grado de instrucción académico de posea la mayor parte de la población. Sin duda esto muestra que la educación en general no es compatible con las demandas de conocimiento y habilidades que los jóvenes demandan. Ellos buscan una educación práctica, flexible, real a las condiciones que requieren los mercados de empleo, porque rechazan la educación conceptual. 


\section{Gráfico 6}

Competencias necesarias para práctica universitaria y profesional

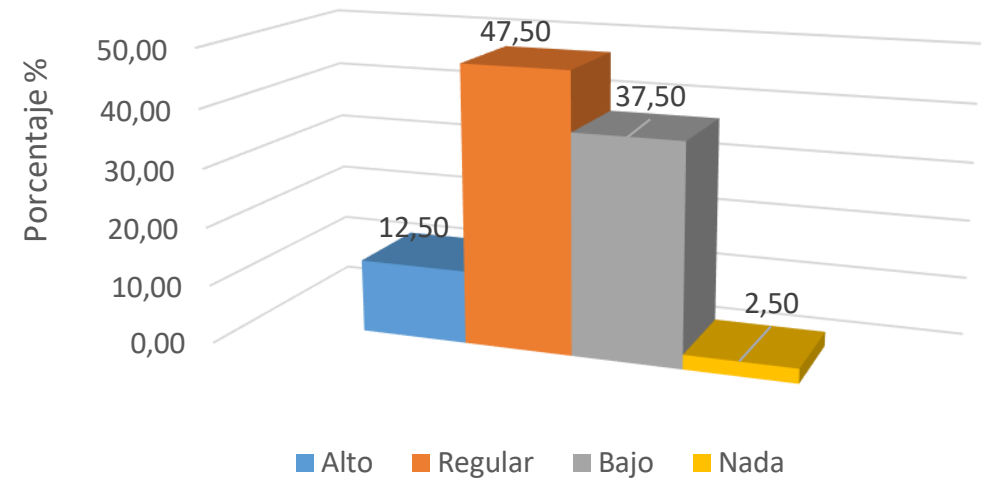

Se observa en la figura 4 que un $47 \%$ y un $38 \%$ de los docentes consideran regular y bajo respectivamente el nivel en que los contenidos y las asignaturas de la educación secundaria son necesarios para generar competencias básicas y necesarias para la práctica universitaria y por ende profesional.

Por tanto, esto debe ir de la mano con la necesidad de adaptar al sistema educativo a un enfoque actual, en donde la educación no sea tan memorística sino que abarque un contexto más acorde a la globalización con el uso de la tecnología para que la información esté al alcance de sus manos y que el valor que añadan las instituciones educativas estén para crear nuevos conocimientos, desarrollar capacidades, competencias, fomentar aptitudes, habilidades para poder transformar realidades, (Olmedo, 2018).

\section{CONCLUSIONES}

El estudio permito determinar que los docentes actuales mantienen prácticas de enseñanza con modelo pedagógico tradicional y los estudiantes aún continúan reteniendo conocimientos de forma también tradicional por repetición y memorística 
en donde el docente cuando imparte su clase, lo realiza en forma de monologo, repitiendo contenidos de un currículo previamente asignado.

Los resultados muestran que a pesar de los avances en la ciencia y en la tecnología, con estudiantes con diferente manera de pensar y actuar a otras épocas, el modelo de pedagogía tradicional todavía está presente en las actividades desarrolladas en clases porque estas no facilitan en forma total la creatividad, se promueve parcialmente la investigación y se usa poco el recurso tecnológico como apoyo al proceso de enseñanza - aprendizaje.

Otro resultado importante es que aunque el Ministerio de Educación de Ecuador pretenda que la educación tenga carácter interdisciplinar por medio de la realización de proyectos que generen en los estudiantes habilidades, destrezas y competencias básicas para la universidad y en lo profesional, la realidad es que está muy lejos de lograrse porque como lo complementa los datos del INEC, solo un $20 \%$ de las personas empleadas tienen un grado de educación superior.

\section{REFERENCIAS CONSULTADAS}

1. Aldana, J. (2019). La competencia epistemológica en el investigador social universitario venezolano. Praxis, 15 (1), 103 -115. Doi: http://dx.doi.org/10.21676/23897856.3091

2. Barragán, T. y Ortega G. (2017). La realidad educativa ecuatoriana desde una perspectiva docente. Revista iberoamericana de educación. 75(2). 9-20

3. Calleja, R. (2010). Un nuevo modelo educativo para el siglo XXI. Comunidad Escolar, periódico digital de información educativa, Número 880, Digital 251, 27 de Octubre. Recuperado de http://comunidadescolar.pntic.ec.es/880/report1.html

4. Constitución de la República del Ecuador. (2008). Constitución del Ecuador. Art. 343. Quito, Ecuador: Registro oficial 449 del 20 de octubre del 2008

5. Dávila (2017). Modelos pedagógicos para la formación docente en ecuador: Una mirada histórica hasta la actualidad. El Pensamiento Educativo Ecuatoriano. 
6. De Zubiría, J. (2006). Los modelos pedagógicos - Hacia una pedagogía dialogante (2a ed.). Bogotá, Colombia: Magisterio. Pag 23

7. De Zubiría, J. (2010). Los Modelos Pedagógicos. Bogotá, Colombia: Magisterio.

8. Delval, J. (2013). La escuela para el siglo XXI. Sinéctica, , (40), 01-18. [Fecha de Consulta 30 de Septiembre de 2019]. ISSN: 1665-109X. Disponible en: https://www.redalyc.org/articulo.oa?id=998/99827467002

9. Educación Ecuador (2018). Listas de carreras universitarias 2019 con mayor demanda en Ecuador. Recuperado de https://educacionecuadorministerio.blogspot.com/2018/02/lista-de-carrerasuniversitarias-ecuador-mas-demandadas.html

10. El Ciudadano (2011). Año de la revolución educativa. El ciudadano.gob.ec (periódico digital del gobierno de la revolución ciudadana), 14 enero del 2011

11. Flórez, R., \& Tobón, A. (2001). Investigación Educativa y Pedagógica. 11(2). Recuperado de http://www.saber.ula.ve/bitstream/handle/123456789/17161/resena.pdf?seque nce $=1$ \&isAllowed $=y$

12. Flórez, R. (1994). Hacia una Pedagogía de Conocimiento. Bogotá, Colombia: McGraw-Hill.

13. Gerver, R. (2010). Crear hoy la escuela del mañana. La educación y el futuro de nuestros hijos. Madrid: SM

14. Gómez, M. (2017). Elementos para la elaboración de un currículo informacional y documental. Revista Latinoamericana de Tecnología Educativa, 16(2), 127141. Recuperado de https://dialnet.unirioja.es/servlet/articulo?codigo $=6244792$

15. INEC, (2018). Tabulados Encuesta Nacional de Empleo, Desempleo y Subempleo (ENEMDU). Disponible en: www.ecuadorencifras.gob.ec

16. Ianni Gómez., L. (2017). Miramda: Una propuesta educativa emergente desde la investigación. Revista Arbitrada Interdisciplinaria Koinonía, 2(3), 9-30. Recuperado https://fundacionkoinonia.com.ve/ojs/index.php/revistakoinonia/article/view/49/ $\underline{36}$

17. López, E. (2011). Las actuales propuestas y desafíos en educación; el caso ecuatoriano. Educación \& Sociedad, 32(115), 373-391 
18. Manzano, J. (2007). Los colegios no deberían existir. Recuperado de http://www.kindsein.com/es/21/1/485/

19. MEE (2016). Currículo de EGB y BGU. Quito: Don Bosco

20. Ministerio de educación del Ecuador (2016). Mineduc. Quito: Don Bosco

21. Murray, S. \& Larry, S. (2009). Estadística. México D.F, México: McGraw-Hill

22. Olmedo. P. (2018). El empleo en el Ecuador. Una mirada a la situación y perspectiva para el mercado laboral actual. Friedrich-Ebert-Stiftung (FES) Ecuador Instituto Latinoamericano de Investigaciones Sociales (ILDIS) QuitoEcuador. Recuperado de https://library.fes.de/pdf-files/bueros/quito/14525.pdf

23. Ortiz, A. (2013). Modelos pedagógicos y teorías del aprendizaje. La Habana, Cuba. https://www.researchgate.net/publicatión/315835198 Modelos Pedagogicos y Teorias del aprendizaje

24. Padilla, K. \& Ortiz, M. (2018). Analisis del modelo pedagógico del centro integral New Genereatión Kids Planet. Tesis de grado. Universidad Politécnica Salesiana Quito. Quito. Ecuador

25. Pozo, J. (2014). Psicología del aprendizaje humano. Madrid: Editorial Morata, S. L.

26. Rius, M. (2012). ¿La escuela mata la creatividad? La Vanguardia, diario matinal de información general. Recuperado de http:/www.lavanguardia.com/estilosde-vida/20120203/54247867713/la-escuela-mata-la-creatividad.html

27. Robinson, K. (2009). El elemento. México: Grijalbo

28. Rodríguez, J. (2013). Una mirada a la pedagogía tradicional y humanista. Presencia Universitaria. 3(5), 36-45

29. Romero, M., \& Villasmil, J. (2017). Repensar la formación docente. hacia el encuentro de una nueva perspectiva epistémica para su abordaje y resignificación. CIENCIAMATRIA, 3(5), 133-149. https://doi.org/10.35381/cm.v3i5.17

30. Toffler, A. (1985). La tercera ola. Barcelona, España: Editorial Orbis

31. UNESCO (2017). La agenda 2030 para el desarrollo sostenible. Declaración de Buenos Aires E2030.Buenos Aires. Argentina 
32. Vásquez, E. L. (2013). Educación y modelos pedagógicos. Recuperado de http://www.boyaca.gov.co/SecEducacion/images/Educ modelos pedag.pdf

33. Vergara, G. y Cuentas, H. (2015). Actual vigencia de los modelos pedagógicos en el contexto educativo. Opción. 31(6). 914-934

\section{REFERENCES CONSULTED}

1. Aldana, J. (2019). Epistemological competence in the Venezuelan university social researcher. Praxis, 15 (1), 103 -115. Doi: http://dx.doi.org/10.21676/23897856.3091

2.

3. Barragán, T. and Ortega G. (2017). The Ecuadorian educational reality from a teaching perspective. Latin American magazine of education. 75 (2). 9-20

4. Calleja, R. (2010). A new educational model for the 21st century. School Community, digital newspaper of educational information, Number 880, Digital 251, October 27. Recovered from http://comunidadescolar.pntic.ec.es/880/report1.html

5. Constitution of the Republic of Ecuador. (2008). Constitution of Ecuador. Art. 343. Quito, Ecuador: Official record 449 of October 20, 2008

6. Dávila (2017). Pedagogical models for teacher training in Ecuador: A historical look to the present. The Ecuadorian Educational Thought. Recovered from http://repositorio.unae.edu.ec/handle/123456789/514

7. De Zubiría, J. (2006). Pedagogical models - Towards a dialogic pedagogy (2nd ed.). Bogotá, Colombia: Teaching. Page 23

8. De Zubiría, J. (2010). The Pedagogical Models. Bogotá, Colombia: Teaching.

9. Delval, J. (2013). The school for the 21st century. Synectics,, (40), 01-18. [Consultation Date September 30, 2019]. ISSN: 1665-109X. Available at: https://www.redalyc.org/articulo.oa?id=998/99827467002

10. Education Ecuador (2018). College race lists 2019 with the highest demand in Ecuador. https://educacionecuadorministerio.blogspot.com/2018/02/lista-de-carrerasuniversitarias-ecuador-mas-demandadas.html

11. The Citizen (2011). Year of the educational revolution. The citizen.gob.ec (digital newspaper of the government of the citizen revolution), January 14, 2011 
12. Flórez, R., \& Tobón, A. (2001). Educational and Pedagogical Research. 11 (2). Retrieved http://www.saber.ula.ve/bitstream/handle/123456789/17161/resena.pdf?seque nce $=1$ \&isAllowed $=y$

13. Flórez, R. (1994). Towards a Pedagogy of Knowledge. Bogota, Colombia: McGraw-Hill.

14. Gerver, R. (2010). Create tomorrow's school today. Education and the future of our children. Madrid: SM

15. Gómez, M. (2017). Elements for the preparation of an informational and documentary curriculum. Latin American Journal of Educational Technology, 16 (2), 127-141. Recovered from https://dialnet.unirioja.es/servlet/articulo?codigo=6244792

16. Ianni Gómez., L. (2017). MIRANDA: An educational proposal emerging from research. Koinonia Interdisciplinary Arbitrated Magazine, 2(3), 9-30. Recuperado de https://fundacionkoinonia.com.ve/ojs/index.php/revistakoinonia/article/view/49/ 36

17. INEC, (2018). Tabulated National Survey of Employment, Unemployment and Under-employment (ENEMDU). Available at: www.ectadorencifras.gob.ec

18. López, E. (2011). Current proposals and challenges in education; The Ecuadorian case. Education \& Society, 32 (115), 373-391

19. Manzano, J. (2007). Schools should not exist. Retrieved from http://www.kindsein.com/es/21/1/485/

20. MEE (2016). EGB and BGU curriculum. Quito: Don Bosco

21. Ministry of Education of Ecuador (2016). Mineduc Quito: Don Bosco

22. Murray, S. \& Larry, S. (2009). Statistics. Mexico D.F, Mexico: McGraw-Hill

23. Olmedo P. (2018). The employment in Ecuador. A look at the situation and perspective for the current labor market. Friedrich-Ebert-Stiftung (FES) Ecuador Latin American Institute for Social Research (ILDIS) Quito-Ecuador. Recovered from https://library.fes.de/pdf-files/bueros/quito/14525.pdf

24. Ortiz, A. (2013). Pedagogical models and learning theories. Havana Cuba. Recovered from 
https://www.researchgate.net/publicatión/315835198_Modelos_Pedagogicos_ y_Teorias_del_aprendizaje

25.Padilla, K. \& Ortiz, M. (2018). Pedagogical model analysis of the New Genereatión Kids Planet integral center. Thesis. Salesian Polytechnic University Quito. Quito Ecuador

26. Pozo, J. (2014). Psychology of human learning. Madrid: Editorial Morata, S. L.

27. Rius, M. (2012). School kills creativity?. La Vanguardia, morning newspaper of general information. Retrieved from http://www.lavanguardia.com/estilos-devida/20120203/54247867713/la-escuela-mata-la-creatividad.html

28. Robinson, K. (2009). The element. Mexico: Grijalbo

29. Romero, M., \& Villasmil, J. (2017). Rethink teacher training. Towards the meeting of a new epistemic perspective for its approach and resignification. CIENCIAMATRIA, 3(5), 133-149. https://doi.org/10.35381/cm.v3i5.17

30. Rodríguez, J. (2013). A look at traditional and humanistic pedagogy. University Presence 3 (5), 36-45

31. Toffler, A. (1985). The third wave Barcelona, Spain: Orbis Editorial

32. UNESCO (2017). The 2030 agenda for sustainable development. Declaration of Buenos Aires E2030. Buenos Aires. Argentina

33. Vásquez, E. L. (2013). Education and pedagogical models. Recovered from http://www.boyaca.gov.co/SecEducacion/images/Educ_modelos_pedag.pdf

34. Vergara, G. and Accounts, H. (2015). Current validity of pedagogical models in the educational context. Option. 31 (6). 914-934 